\title{
SOME DIFFERENTIAL OPERATORS IN THE SYMMETRIC BUNDLE
}

\author{
Anna Kimaczyńska \\ Institute of Mathematics and Computer Science, University of Lodz \\ Lodz, Poland \\ kimaczynska@math.uni.lodz.pl
}

Received: 12 June 2017; Accepted: 11 September 2017

\begin{abstract}
Some natural differential operators in the bundles of symmetric tensors and symmetric tensors with values in the tangent bundle are investigated. Applications in geometry, physics and tomography are also reviewed.
\end{abstract}

MSC 2010: 58C05, 47F05, 53C21

Keywords: symmetric tensors, symmetric derivative, the gradient, the divergence, Weitzenböck type formula

\section{Introduction}

Differential operators in the bundles of symmetric tensors and symmetric tensors with values in the tangent bundle on a Riemannian manifold are more and more often a subject of interest of contemporary geometry. Recently, such operators were investigated e.g. in [1], by Balcerzak and Pierzchalski in the category of Lie algebroids or, in [2], by Stepanov and Mikes, in the case of one tensors where some spectral properties of the Yano rough Laplacian - the operator similar to the one considered here Sampson Laplacian $\Delta^{\mathrm{s}}$ - were analyzed. It is also worth noticing a very recent paper [3], by Heil, Moroianu and Semmelmann where some elliptic operators in the bundle of symmetric forms were investigated in the context of Killing and conformal Killing tensors. In this context the symmetric tensors were also investigated earlier in [4].

The operators of gradient and the divergence on symmetric tensors were investigated in detail in the author's recent $\mathrm{PhD}$ dissertation [5]. Their boundary behavior when $M$ is a manifold with a nonempty boundary was investigated in [6].

One of the most important operators acting on symmetric tensors is the first order linear operator $\mathrm{d}^{\mathrm{s}}$ (defined by (7)) being the symmetric part of the Levi-Civita covariant derivative $\nabla$. In addition to $\mathrm{d}^{\mathrm{s}}$, we consider three other zero order operators: $a$ (defined by (13)) and two traces Tr and tr (defined by (11) and (12)), respec- 
tively). It is interesting that linear combinations of the mentioned four operators and their adjoints led to several interesting operators. Their adjoints led to several interesting operators.

First of all, let us consider the two first order differential operators grad $=$ $=a \mathrm{~d}^{\mathrm{s}}-\mathrm{d}^{\mathrm{s}} a$ and div $=\operatorname{Tr} \mathrm{d}^{\mathrm{s}}-\mathrm{d}^{\mathrm{s}} \operatorname{Tr}$. They are adjointed - each to the other - with respect to the global (integral) scalar product on $M$ (cf. Proposition 2.5). They reduce to the usual gradient when acting on functions and the usual divergence when acting on vector fields.

The main result of our paper is Theorem 2.3. It states that in fact our operator div grad is the negative of the classical Bochner-Laplace operator: $-\operatorname{tr} \nabla \nabla$. We also consider the operator $\Delta^{\mathrm{s}}=\mathrm{d}^{\mathrm{s}^{*}} \mathrm{~d}^{\mathrm{s}}-\mathrm{d}^{\mathrm{s}} \mathrm{d}^{\mathrm{s}^{*}}$ which was considered first by J.H. Sampson and investigated in the context of a Chern theorem in [7]. Both $-\operatorname{tr} \nabla \nabla$ and $\Delta^{\mathrm{s}}$ are second order differential operators. They are both strongly elliptic in the sense that their symbols are positively defined (cf. [6]).

It is interesting that, similar to analogous operators in the case of skewsymmetric tensors, the difference between the negative of div grad and $\Delta^{\mathrm{s}}$ is a zero order operator (tensor) depending on the curvature, i.e. a Weitzenböck type formula holds in the bundle of symmetric tensors (cf. Theorem 3.1). On the other hand, the formula relating div grad to the Bochner Laplacian in Theorem 2.3 gives an equivalence of our Weitzenböck type formula (21) to the classical Weitzenböck formula (22) proved e.g. in [5] or in [7].

Finally, we discuss some possible applications in geometry, physics and tomography.

The author would like to express her gratitude to Antoni Pierzchalski for suggesting the problem and discussions.

\section{Natural differential operators in the bundle of symmetric tensors}

All the objects and morphisms are assumed to be smooth, i.e. of class $C^{\infty}$.

Let $(M, \mathrm{~g})$ be an oriented Riemannian manifold of dimension $n$. Denote by $C^{\infty}(M)$ the ring of smooth functions on $M$. Let $T=T M$ and $T^{*}=T^{*} M$ be the tangent and cotangent bundles, respectively. Denote by $T^{* k}=T^{* k} M$ the bundle of $k$-tensors on $M$ and by $S^{k}=S^{k} M$ its subbundle of $k$-symmetric tensors ( $k$-forms). For any bundle $E$ over $M$ denote by $C^{\infty}(E)$ the $C^{\infty}(M)$ - module of sections of $E$.

For any $\mathrm{p} \in M$, g defines a scalar product in $\mathrm{T}_{\mathrm{p}}$ :

$$
\langle\cdot, \cdot\rangle=\mathrm{g}_{\mathrm{p}}(\cdot, \cdot): \mathrm{T}_{\mathrm{p}} \times \mathrm{T}_{\mathrm{p}} \rightarrow \mathrm{R} .
$$

Extend $\langle\cdot, \cdot\rangle$ in a natural way to the fibers of the cotangent bundle and next to the fibers of any tensor bundle on M. In particular, the bundle of symmetric tensors $S^{k}$ as a subbundle of $T^{* k}$ inherits this scalar product. Consider also in this bundle another scalar product 


$$
\langle\cdot \mid \cdot\rangle=1 / \mathrm{k} !\langle\cdot, \cdot\rangle
$$

Let $\nabla$ be the Levi-Civita covariant derivative of the metric $g$ on $M$. Trasmit $\nabla$ in a natural way from the tangent bundle $T$ to the dual bundle $T^{*}\left(=S^{1}\right)$ and next to any tensor bundle by the Leibniz rule, in particular to the bundle $S^{k}$. The extended connection is denoted by the same symbol $\nabla$.

If, for $\varphi \in C^{\infty}\left(S^{k}\right)$ we use the convention:

$$
(\nabla \varphi)\left(X, X_{1}, \ldots X_{k}\right)=\left(\nabla_{X} \varphi\right)\left(X_{1}, \ldots X_{k}\right),
$$

the obtained covariant derivative may be treated as the map:

$$
\nabla: C^{\infty}\left(S^{k}\right) \rightarrow C^{\infty}\left(T^{*} \otimes S^{k}\right)
$$

For any $1 \leq \mathrm{i}, \mathrm{j}, \mathrm{r} \leq \mathrm{n}$ and any local frame $\mathrm{e}_{1}, \ldots, \mathrm{e}_{\mathrm{n}}$ on $M$ define the Christoffel symbols $\Gamma_{\mathrm{jr}}^{\mathrm{i}}$ by

$$
\nabla_{\mathrm{e}_{\mathrm{j}}} \mathrm{e}_{\mathrm{r}}=\sum_{\mathrm{i}=1, \ldots, \mathrm{n}} \Gamma_{\mathrm{jr}}^{\mathrm{i}} \mathrm{e}_{\mathrm{i}} .
$$

One can easily prove that if $\mathrm{e}_{1}, \ldots, \mathrm{e}_{\mathrm{n}}$ is a local orthonormal frame on $M$ then for any $1 \leq \mathrm{i}, \mathrm{j}, \mathrm{r} \leq \mathrm{n}$

$$
\Gamma_{\mathrm{jr}}^{\mathrm{i}}=-\Gamma_{\mathrm{ji}}^{\mathrm{r}} .
$$

If $E$ is any vector bundle over $M$ and $\langle\cdot, \cdot\rangle$ is any scalar product in $E$, the global scalar product $(\cdot, \cdot)$ in the space of sections of $E$ can be defined by

$$
(\cdot, \cdot)=\int_{M}\langle\cdot \cdot \cdot\rangle \Omega_{M},
$$

where $\Omega_{M}$ is the volume form on $M$ defined by the orientation and the metric $\mathrm{g}$ (cf. [8]).

The global scalar product is then well defined only for such pairs of sections that the integral exists and is finite. This is always the case when, e.g. at least one of the sections is of compact support.

That way, for the bundle $S^{k}$, we have two global scalar products $(\cdot, \cdot)$ and $(\cdot \cdot \cdot)$. They (cf. (1)) are related by

$$
(\cdot \mid \cdot)=1 / \mathrm{k} !(\cdot, \cdot) \cdot
$$

Define the operator of symmetric derivative

$d^{\mathrm{s}}: C^{\infty}\left(S^{\mathrm{k}}\right) \rightarrow C^{\infty}\left(S^{\mathrm{k}+1}\right)$

by

$$
\mathrm{d}^{\mathrm{s}}=(\mathrm{k}+1) \operatorname{Sym} \nabla,
$$

where Sym: $T^{* k} \rightarrow T^{* k}$ is the linear operation of symmetrization: 


$$
(\operatorname{Sym} \psi)\left(X_{1}, \ldots, X_{k}\right)=1 \backslash k ! \sum_{\sigma} \psi\left(X_{\sigma(1)}, \ldots X_{\sigma(k)}\right),
$$

$\mathrm{X}_{1}, \ldots, \mathrm{X}_{\mathrm{k}} \in T$.

A local expression for the symmetric derivative by the covariant derivative is the following

Proposition 2.1. Let $\mathrm{e}_{1}, \ldots, \mathrm{e}_{\mathrm{n}}$ be a local frame of sections of $\mathrm{T}$ and let $\mathrm{e}_{1}{ }^{*}, \ldots, \mathrm{e}_{\mathrm{n}}{ }^{*}$ be the dual frame then

$$
\mathrm{d}^{\mathrm{s}} \varphi=\sum_{\mathrm{j}=1, \ldots, \mathrm{n}} \mathrm{e}_{\mathrm{j}}^{*} \odot \nabla_{\mathrm{e}_{\mathrm{j}}} \varphi,
$$

for $\varphi \in C^{\infty}\left(S^{\mathrm{k}}\right)$.

Proof. The proof is analogous to that one for local expression of the exterior derivative in the bundle of skew-symmetric tensors given e.g. in [9].

Define the operator $t_{\mathrm{x}}$ of substitution of $\mathrm{X} \in C^{\infty}(T)$ as the mapping $\imath_{\mathrm{X}} C^{\infty}\left(S^{\mathrm{k}}\right) \rightarrow\left(S^{\mathrm{k}-1}\right)$ of form

$$
\left(\mathrm{l}_{\mathrm{X}} \varphi\right)\left(\mathrm{X}_{1}, \ldots, \mathrm{X}_{\mathrm{k}-1}\right)=\varphi\left(\mathrm{X}, \mathrm{X}_{1}, \ldots, \mathrm{X}_{\mathrm{k}-1}\right), \text { for } \mathrm{k}>0
$$

and

$$
\mathrm{t}_{\mathrm{X}} \varphi=0, \text { for } \mathrm{k}=0
$$

where $\mathrm{X}_{1}, \ldots, \mathrm{X}_{\mathrm{k}-1} \in C^{\infty}(T), \varphi \in C^{\infty}\left(S^{\mathrm{k}}\right)$.

One can also easily prove that, for $\mathrm{X}, \mathrm{Y} \in C^{\infty}(T)$.

$$
\mathfrak{l}_{\mathrm{X}} \nabla_{\mathrm{Y}}=\nabla_{\mathrm{Y}} \mathrm{l}_{\mathrm{X}}-\mathfrak{\imath}_{\mathrm{Vx} \mathrm{Y}}
$$

Extend the symmetric derivative to

$\mathrm{d}^{\mathrm{s}}: C^{\infty}\left(S^{\mathrm{k}} \otimes T\right) \rightarrow C^{\infty}\left(S^{\mathrm{k}+1} \otimes T\right)$

by the formula

$$
\mathrm{d}^{\mathrm{s}}(\varphi \otimes \mathrm{X})=\mathrm{d}^{\mathrm{s}} \varphi \otimes \mathrm{X}+\varphi \odot \nabla \mathrm{X},
$$

for $\varphi \otimes \mathrm{X} \in C^{\infty}\left(S^{\mathrm{k}} \otimes T\right)$ where $\nabla \mathrm{X}$ is treated as 1-form with values in $T$. Locally this form can be given by $\nabla \mathrm{X}=\sum_{\mathrm{j}=1, \ldots, \mathrm{n}} \mathrm{e}_{\mathrm{j}}^{*} \otimes \nabla_{\mathrm{e}_{\mathrm{j}}} \mathrm{X}$.

In analogy to Proposition 2.1, one can prove

Proposition 2.2. Let $\mathrm{e}_{1}, \ldots, \mathrm{e}_{\mathrm{n}}$ be a local frame of sections of $\mathrm{T}$ and let $\mathrm{e}_{1}{ }^{*}, \ldots, \mathrm{e}_{\mathrm{n}}{ }^{*}$ be the dual frame then

$$
\mathrm{d}^{\mathrm{s}} \Phi=\sum_{\mathrm{j}=1, \ldots, \mathrm{n}} \mathrm{e}_{\mathrm{j}}^{*} \odot \nabla_{\mathrm{e}_{\mathrm{j}}} \Phi
$$

for $\Phi \in C^{\infty}\left(S^{\mathrm{k}} \otimes T\right)$.

Define now two trace operators. First, the trace operator 
$\operatorname{Tr}: C^{\infty}\left(T^{* \mathrm{k}} \otimes T\right) \rightarrow C^{\infty}\left(T^{* \mathrm{k}-1}\right)$

acting on vector forms $\varphi \otimes \mathrm{X} \in C^{\infty}\left(T^{* \mathrm{k}} \otimes T\right)$ by

$$
\operatorname{Tr}(\varphi \otimes X)=\mathfrak{l}_{\mathrm{X}} \varphi, \text { for } \mathrm{k} \geq 0 .
$$

Next, the trace operator

$$
\operatorname{tr}: C^{\infty}\left(T^{* \mathrm{k}}\right) \rightarrow C^{\infty}\left(T^{* \mathrm{k}-2}\right)
$$

defined by the metric $g$ and acting on scalar forms $\varphi \in C^{\infty}\left(T^{* \mathrm{k}}\right)$ by

$$
\operatorname{tr} \varphi=\sum_{\mathrm{i}=1, \ldots, \mathrm{n}} \mathrm{e}_{\mathrm{i}} \mathrm{e}_{\mathrm{i}} \varphi, \text { for } \mathrm{k} \geq 0 .
$$

Here $\mathrm{e}_{1}, \ldots, \mathrm{e}_{\mathrm{n}}$ is an orthonormal frame of $T$.

The right hand side of (12) is independent of the choice of frame. We will use the same symbols for the restrictions of operators $\operatorname{Tr}$ and tr to the subbundles $S^{\mathrm{k}} \otimes T$ and $S^{\mathrm{k}}$, of the bundles $T^{* \mathrm{k}}$ and $T^{* \mathrm{k}} \otimes T$, respectively.

Now, we are ready to get the shape of operators formally adjoint to $\mathrm{d}^{\mathrm{s}}$.

Theorem 2.1. With respect to the global scalar product $(\cdot \cdot)$ the operator $d^{t^{*}}$ : $\mathrm{C}^{\infty}\left(\mathrm{S}^{\mathrm{k}+1}\right) \rightarrow \mathrm{C}^{\infty}\left(\mathrm{S}^{\mathrm{k}}\right)$ formally adjoint to $d^{\mathrm{s}}: \mathrm{C}^{\infty}\left(\mathrm{S}^{\mathrm{k}}\right) \rightarrow \mathrm{C}^{\infty}\left(\mathrm{S}^{\mathrm{k}+1}\right)$ is of form

$$
\mathrm{d}^{\mathrm{s}^{*}}=-\operatorname{tr} \nabla_{\mid C^{\infty}}\left(S^{k+1}\right) \text {. }
$$

Proof. See e.g. [6].

Theorem 2.2. With respect to the global scalar product $(\cdot \mid \cdot)$ the operator $d^{S *}$ : $\mathrm{C}^{\infty}\left(\mathrm{S}^{\mathrm{k}+1} \otimes \mathrm{T}\right) \rightarrow \mathrm{C}^{\infty}\left(\mathrm{S}^{\mathrm{k}} \otimes \mathrm{T}\right)$ formally adjoint to $d^{\mathrm{S}}: \mathrm{C}^{\infty}\left(\mathrm{S}^{\mathrm{k}} \otimes \mathrm{T}\right) \rightarrow \mathrm{C}^{\infty}\left(\mathrm{S}^{\mathrm{k}+1} \otimes \mathrm{T}\right)$ is of form

$$
\mathrm{d}^{\mathrm{s}^{*}}=-\operatorname{tr} \nabla_{\mid \mathrm{C}^{\infty}\left(\mathrm{S}^{\mathrm{k}+1} \otimes \mathrm{T}\right)}
$$

Proof. See e.g. [5].

Next, for $k=0,1, \ldots$ define the operator $a: C^{\infty}\left(S^{k}\right) \rightarrow C^{\infty}\left(S^{k-1} \otimes T\right)$ by

$$
a \varphi=\sum_{\mathrm{i}=1, \ldots, \mathrm{n}} \mathrm{le}_{\mathrm{i}} \varphi \otimes \mathrm{e}_{\mathrm{i}}
$$

where $\mathrm{e}_{1}, \ldots, \mathrm{e}_{\mathrm{n}}$ is an orthonormal basis in $T$ and $\varphi \in C^{\infty}\left(S^{\mathrm{k}}\right)$.

One can easily see that the definition of $a$ is independent of the choice of orthonormal frame.

Define now two differential operators: the gradient acting on symmetric tensors of any degree and the divergence acting on vector valued symmetric tensors of any degree.

The gradient is the operator

$$
\text { grad: } C^{\infty}\left(S^{\mathrm{k}}\right) \rightarrow C^{\infty}\left(S^{\mathrm{k}} \otimes T\right)
$$


defined by

$$
\operatorname{grad}=a \mathrm{~d}^{\mathrm{s}}-\mathrm{d}^{\mathrm{s}} a
$$

Its local shape is expressed by

Proposition 2.3. Let $\mathrm{e}_{1}, \ldots, \mathrm{e}_{\mathrm{n}}$ be a local orthonormal frame of sections of $\mathrm{T}$ then locally

$$
\operatorname{grad} \varphi=\sum_{\mathrm{i}=1, \ldots, \mathrm{n}} \nabla_{\mathrm{e}_{\mathrm{i}}} \varphi \otimes \mathrm{e}_{\mathrm{i}}
$$

for $\varphi \in \mathrm{C}^{\infty}\left(S^{\mathrm{k}}\right)$.

Proof. See [5].

The divergence is the operator

$$
\operatorname{div}: C^{\infty}\left(S^{\mathrm{k}} \otimes T\right) \rightarrow \mathrm{C}^{\infty}\left(S^{\mathrm{k}}\right)
$$

defined by

$$
\operatorname{div}=\operatorname{Tr} d^{\mathrm{s}}-\mathrm{d}^{\mathrm{s}} \operatorname{Tr}
$$

Its local shape is expressed by

Proposition 2.4. For $\varphi \otimes \mathrm{X} \in C^{\infty}\left(S^{k} \otimes T\right)$ we have

$$
\operatorname{div}(\varphi \otimes X)=\nabla_{X} \varphi+\varphi \operatorname{div} X
$$

where in any local orthonormal basis $\mathrm{e}_{1}, \ldots, \mathrm{e}_{\mathrm{n}}$ the $\operatorname{div} \mathrm{X}$ is defined locally by

$$
\operatorname{div} \mathrm{X}=\sum_{\mathrm{j}=1, \ldots, \mathrm{n}}\left\langle\mathrm{e}_{\mathrm{j}}, \nabla_{\mathrm{e}_{\mathrm{j}}} \mathrm{X}\right\rangle .
$$

Proof. See [5].

Proposition 2.5. The differential operators - grad: $\mathrm{C}^{\infty}\left(\mathrm{S}^{\mathrm{k}}\right) \rightarrow \mathrm{C}^{\infty}\left(\mathrm{S}^{\mathrm{k}} \otimes \mathrm{T}\right)$ and $\operatorname{div}: \mathrm{C}^{\infty}\left(\mathrm{S}^{\mathrm{k}} \otimes \mathrm{T}\right) \rightarrow \mathrm{C}^{\infty}\left(\mathrm{S}^{\mathrm{k}}\right)$ are formally adjoint (each to the other) with respect to the global scalar product $(\cdot \mid \cdot)$.

Proof. See [6].

Consider now the composition of our two operators div and grad, i.e. the second order operator

$$
\operatorname{div} \operatorname{grad}: C^{\infty}\left(S^{\mathrm{k}}\right) \rightarrow C^{\infty}\left(S^{\mathrm{k}}\right) .
$$

For any $\mathrm{X}, \mathrm{Y} \in C^{\infty}(T)$ define the second order derivative $\nabla_{\mathrm{X}, \mathrm{Y}}^{2}$ by

$$
\nabla_{X, Y}^{2}=\nabla_{X} \nabla_{Y}-\nabla_{\nabla x Y}
$$

The classical Bochner-Laplace operator $-\operatorname{tr} \nabla \nabla$ is related to the second order derivative: 
Proposition 2.6. In any local orthonormal frame $\mathrm{e}_{1}, \ldots, \mathrm{e}_{\mathrm{n}}$ on $M$

$$
-\operatorname{tr} \nabla \nabla=-\sum_{\mathrm{i}=1, \ldots, \mathrm{n}} \nabla^{2} \mathrm{e}_{\mathrm{i}, \mathrm{e}_{\mathrm{i}}} .
$$

Proof. See [5].

Let us prove the main result of the paper saying that on the symmetric tensors the operator div grad coincides with the negative of the classical Bochner-Laplace operator:

\section{Theorem 2.3.}

$$
\operatorname{div} \operatorname{grad}=\operatorname{tr} \nabla \nabla
$$

Proof. Let $\varphi \in C^{\infty}\left(S^{\mathrm{k}}\right)$. By Proposition 2.3 and Proposition 2.4 we have locally

$$
\begin{gathered}
\operatorname{div} \operatorname{grad} \varphi=\operatorname{div}\left(\sum_{\mathrm{i}=1, \ldots, \mathrm{n}} \nabla_{\mathrm{e}_{\mathrm{i}}} \varphi \otimes \mathrm{e}_{\mathrm{i}}\right) \\
=\sum_{\mathrm{i}=1, \ldots, \mathrm{n}}\left(\nabla \mathrm{e}_{\mathrm{e}} \nabla_{\mathrm{e}_{\mathrm{i}}} \varphi+\nabla \mathrm{e}_{\mathrm{i}} \varphi\left(\sum_{\mathrm{j}=1, \ldots, \mathrm{n}}\left\langle\mathrm{e}_{\mathrm{j}}, \nabla_{\mathrm{e}_{\mathrm{j}}} \mathrm{e}_{\mathrm{i}}\right\rangle\right)\right),
\end{gathered}
$$

where $\mathrm{e}_{1}, \ldots, \mathrm{e}_{\mathrm{n}}$ is a local orthonormal frame on $M$. So, by the definition of Christoffel symbols and (4) we can continue sequentially with

$$
\begin{aligned}
& \sum_{\mathrm{i}=1, \ldots, \mathrm{n}} \nabla_{\mathrm{e}_{\mathrm{i}}} \nabla_{\mathrm{e}_{\mathrm{i}}} \varphi+\sum_{\mathrm{i} \mathrm{j}=1, \ldots, \mathrm{n}} \Gamma_{\mathrm{ji}}^{\mathrm{j}} \nabla_{\mathrm{e}_{\mathrm{i}}} \varphi \\
= & \sum_{\mathrm{i}=1, \ldots, \mathrm{n}} \nabla_{\mathrm{e}_{\mathrm{i}}} \nabla_{\mathrm{e}_{\mathrm{i}}} \varphi-\sum_{\mathrm{i}, \mathrm{j}=1, \ldots, \mathrm{n}} \Gamma_{\mathrm{j} j}^{\mathrm{i}} \nabla_{\mathrm{e}_{\mathrm{i}}} \varphi \\
= & \sum_{\mathrm{j}=1, \ldots, \mathrm{n}} \nabla_{\mathrm{e}_{\mathrm{j}}} \nabla_{\mathrm{e}_{\mathrm{j}}} \varphi-\sum_{\mathrm{j}=1, \ldots, \mathrm{n}} \nabla \nabla \mathrm{e}_{\mathrm{j}} \mathrm{e}_{\mathrm{j}} \varphi .
\end{aligned}
$$

By (18) and Proposition 2.6 we get the assertion.

\section{Weitzenböck formula for div grad operator}

Let us start with the following:

Definition 3.1. The Laplace operator $\Delta^{s}: C^{\infty}\left(S^{k}\right) \rightarrow C^{\infty}\left(S^{k}\right)$ is the second order differential operator of form:

$$
\Delta^{\mathrm{s}}=\mathrm{d}^{\mathrm{s}^{*}} \mathrm{~d}^{\mathrm{s}}-\mathrm{d}^{\mathrm{s}} \mathrm{d}^{\mathrm{s}^{*}}
$$

The operator $\Delta^{\mathrm{s}}$ was introduced first by Sampson in [7]. Recently this operator has been investigated in the category of Lee algebroids in [1]. For $k=1$ in [2] a similar operator: the Yano rough Laplacian was analyzed in a context of its spectral properties. Some elliptic operators in the bundle of symmetric forms were also investigated in [3] in a context of so-called conformal Killing tensors.

Notice the contrast (in the sign of summands) to the case of the analogous Laplace operator acting in the bundle of skew-symmetric tensors: $\Delta=\mathrm{d}^{*} \mathrm{~d}+\mathrm{d} \mathrm{d}^{*}$, where $d$ is the exterior derivative, or even to the so-called weighted Laplacian: $\Delta_{\mathrm{ab}}=\mathrm{ad}^{*} \mathrm{~d}+\mathrm{bd} \mathrm{d}^{*}$ with constants $a$ and $b$ necessarily positive (investigated e.g. in [10]). 
The aim of this chapter is the discussion of a Weitzenböck type formula. In our case, the formula will relate two differential operators on symmetric forms: - div grad and the Laplace operator defined in (19). Their difference is a zero order operator (tensor) depending on the curvature operator.

The curvature operator is the zero order operator $\mathrm{R}_{\mathrm{X}, \mathrm{Y}}$ defined by

$$
\mathrm{R}_{\mathrm{X}, \mathrm{Y}}=\nabla_{\mathrm{X}, \mathrm{Y}}^{2}-\nabla_{\mathrm{Y}, \mathrm{X}}^{2}
$$

for any $\mathrm{X}, \mathrm{Y} \in C^{\infty}(T)$.

The Ricci type tensor $\Re$ is locally defined by

$$
\mathfrak{R}=\sum_{\mathrm{i}, \mathrm{j}=1, \ldots, \mathrm{n}} \mathrm{e}_{\mathrm{j}}^{*} \odot \mathrm{le}_{\mathrm{i}} \mathrm{Re}_{\mathrm{i}, \mathrm{e}} \mathrm{e}_{\mathrm{j}},
$$

where $\mathrm{e}_{1}, \ldots, \mathrm{e}_{\mathrm{n}}$ is a local orthonormal frame on $M$ and $\mathrm{e}_{1}{ }^{*}, \ldots, \mathrm{e}_{\mathrm{n}}{ }^{*}$ is the dual frame.

One can easily see that the right hand side of (20) is independent of the choice of frames.

Now we are ready to prove the following Weitzenböck type formula for our operator - div grad.

Theorem 3.1 (Weitzenböck type formula) The following formula holds

$$
\Delta^{\mathrm{s}}=-\operatorname{div} \operatorname{grad}-\mathfrak{R} .
$$

Proof. Let $\mathrm{e}_{1}, \ldots, \mathrm{e}_{\mathrm{n}}$ be a local orthonormal frame on $M$ and $\mathrm{e}_{1}{ }^{*}, \ldots, \mathrm{e}_{\mathrm{n}}{ }^{*}$ be the dual frame. By the definition of $\Delta^{\mathrm{s}}$, the shape of $\mathrm{d}^{\mathrm{s}^{*}}$ and $\mathrm{d}^{\mathrm{s}},(8)$ and (4) we have sequentially:

$$
\begin{aligned}
& \Delta^{\mathrm{s}} \varphi=\mathrm{d}^{\mathrm{s}^{*}} \mathrm{~d}^{\mathrm{s}} \varphi-\mathrm{d}^{\mathrm{s}} \mathrm{d}^{\mathrm{s}^{*}} \varphi=-\operatorname{tr} \nabla\left(\sum_{\mathrm{j}=1, \ldots, \mathrm{n}} \mathrm{e}_{\mathrm{j}}{ }^{*} \odot \nabla_{\mathrm{e}_{\mathrm{j}}} \varphi\right)+\sum_{\mathrm{j}=1, \ldots, \mathrm{n}} \mathrm{e}_{\mathrm{j}}{ }^{*} \odot \nabla_{\mathrm{e}_{\mathrm{j}}} \operatorname{tr} \nabla \varphi \\
& =-\sum_{\mathrm{i}, \mathrm{j}=1, \ldots, \mathrm{n}} \mathrm{le}_{\mathrm{i}} \nabla_{\mathrm{e}_{\mathrm{i}}}\left(\mathrm{e}_{\mathrm{j}}{ }^{*} \odot \nabla_{\mathrm{e}_{\mathrm{j}}} \varphi\right)+\sum_{\mathrm{i}, \mathrm{j}=1, \ldots, \mathrm{n}} \mathrm{e}_{\mathrm{j}}{ }^{*} \odot \nabla_{\mathrm{e}_{\mathrm{j}}} \mathrm{e}_{\mathrm{i}} \nabla_{\mathrm{e}_{\mathrm{i}}} \varphi \\
& =-\sum_{\mathrm{i}, \mathrm{j}=1, \ldots, \mathrm{n}} \mathrm{le}_{\mathrm{i}}\left(\nabla_{\mathrm{e}_{\mathrm{i}}} \mathrm{e}_{\mathrm{j}}{ }^{*} \odot \nabla_{\mathrm{e}_{\mathrm{j}}} \varphi\right)-\sum_{\mathrm{i}, \mathrm{j}=1, \ldots, \mathrm{n}} \mathrm{e}_{\mathrm{i}}\left(\mathrm{e}_{\mathrm{j}}{ }^{*} \odot \nabla \mathrm{e}_{\mathrm{i}} \nabla_{\mathrm{e}_{\mathrm{j}}} \varphi\right) \\
& +\sum_{\mathrm{i}, \mathrm{j}=1, \ldots, \mathrm{n}} \mathrm{e}_{\mathrm{j}}{ }^{*} \odot \mathrm{re}_{\mathrm{i}} \nabla_{\mathrm{e}_{\mathrm{j}}} \nabla_{\mathrm{e}_{\mathrm{i}}} \varphi+\sum_{\mathrm{i}, \mathrm{j}=1, \ldots, \mathrm{n}} \mathrm{e}_{\mathrm{j}}{ }^{*} \odot_{\mathrm{v}_{\nabla \mathrm{jei}}} \nabla_{\mathrm{e}_{\mathrm{i}}} \varphi \\
& =-\sum_{\mathrm{i}, \mathrm{j}=1, \ldots, \mathrm{n}} \mathrm{le}_{\mathrm{i}} \nabla \mathrm{e}_{\mathrm{i}} \mathrm{e}_{\mathrm{j}}{ }^{*} \odot \nabla \mathrm{e}_{\mathrm{j}} \varphi-\sum_{\mathrm{i}, \mathrm{j}=1, \ldots, \mathrm{n}} \nabla_{\mathrm{e}_{\mathrm{i}}} \mathrm{e}_{\mathrm{j}}{ }^{*} \odot \mathrm{ue}_{\mathrm{i}} \nabla \mathrm{e}_{\mathrm{j}} \varphi \\
& -\sum_{\mathrm{i}, \mathrm{j}=1, \ldots, \mathrm{n}} \mathrm{e}_{\mathrm{e}_{\mathrm{j}}} \mathrm{e}_{\mathrm{j}}^{*} \odot \nabla \mathrm{e}_{\mathrm{i}} \nabla_{\mathrm{e}_{\mathrm{j}}} \varphi-\sum_{\mathrm{i}, \mathrm{j}=1, \ldots, \mathrm{n}} \mathrm{e}_{\mathrm{j}}{ }^{*} \odot \mathrm{e}_{\mathrm{i}} \nabla_{\mathrm{e}_{\mathrm{i}}} \nabla_{\mathrm{e}_{\mathrm{j}}} \varphi \\
& +\sum_{\mathrm{i}, \mathrm{j}=1, \ldots, \mathrm{n}} \mathrm{e}_{\mathrm{j}}^{*} \odot \mathrm{le}_{\mathrm{i}} \nabla_{\mathrm{e}_{\mathrm{j}}} \nabla \mathrm{e}_{\mathrm{i}} \varphi+\sum_{\mathrm{i}, \mathrm{j}, \mathrm{r}=1, \ldots, \mathrm{n}} \mathrm{e}_{\mathrm{j}}^{*} \odot \Gamma_{\mathrm{ji}}^{\mathrm{r}} \mathrm{e}_{\mathrm{r}} \nabla_{\mathrm{e}_{\mathrm{i}}} \varphi \\
& =-\sum_{\mathrm{i}, \mathrm{j}, \mathrm{r}=1, \ldots, \mathrm{n}} \Gamma_{\mathrm{ij}}^{\mathrm{r}} \mathrm{e}_{\mathrm{i}} \mathrm{e}_{\mathrm{r}}{ }^{*} \odot \nabla_{\mathrm{e}_{\mathrm{j}}} \varphi-\sum_{\mathrm{i}, \mathrm{j}, \mathrm{r}=1, \ldots, \mathrm{n}} \Gamma_{\mathrm{ij}}^{\mathrm{r}} \mathrm{e}_{\mathrm{r}}{ }^{*} \odot \mathrm{le}_{\mathrm{i}} \nabla_{\mathrm{e}_{\mathrm{j}}} \varphi \\
& -\sum_{\mathrm{i}, \mathrm{j}=1, \ldots, \mathrm{n}} \delta_{\mathrm{j}}^{\mathrm{i}} \nabla_{\mathrm{e}_{\mathrm{i}}} \nabla_{\mathrm{e}_{\mathrm{j}}} \varphi-\sum_{\mathrm{i}, \mathrm{j}=1, \ldots, \mathrm{n}} \mathrm{e}_{\mathrm{j}}{ }^{*} \odot \mathrm{le}_{\mathrm{i}} \nabla_{\mathrm{e}_{\mathrm{i}}} \nabla_{\mathrm{e}_{\mathrm{j}}} \varphi \\
& +\sum_{\mathrm{i}, \mathrm{j}=1, \ldots, \mathrm{n}} \mathrm{e}_{\mathrm{j}}^{*} \odot \mathrm{e}_{\mathrm{i}} \nabla_{\mathrm{e}_{\mathrm{j}}} \nabla_{\mathrm{e}_{\mathrm{i}}} \varphi-\sum_{\mathrm{i}, \mathrm{j}, \mathrm{r}=1, \ldots, \mathrm{n}} \mathrm{e}_{\mathrm{j}}{ }^{*} \odot \Gamma_{\mathrm{jr}}^{\mathrm{i}} \mathrm{e}_{\mathrm{r}} \nabla_{\mathrm{e}_{\mathrm{i}}} \varphi \\
& =-\sum_{\mathrm{i}, \mathrm{j}, \mathrm{r}=1, \ldots, \mathrm{n}} \Gamma_{\mathrm{ij}}^{\mathrm{r}} \delta_{\mathrm{r}}^{\mathrm{i}} \nabla_{\mathrm{e}_{\mathrm{j}}} \varphi+\sum_{\mathrm{i}, \mathrm{j}, \mathrm{r}=1, \ldots, \mathrm{n}} \Gamma_{\mathrm{ir}}^{\mathrm{j}} \mathrm{e}_{\mathrm{r}}{ }^{*} \odot_{\mathrm{e}_{\mathrm{i}}} \nabla_{\mathrm{e}_{\mathrm{j}}} \varphi \\
& -\sum_{\mathrm{i}=1, \ldots, \mathrm{n}} \nabla \mathrm{e}_{\mathrm{i}} \nabla_{\mathrm{e}_{\mathrm{i}}} \varphi-\sum_{\mathrm{i}, \mathrm{j}=1, \ldots, \mathrm{n}} \mathrm{e}_{\mathrm{j}}{ }^{*} \bigodot_{\mathrm{e}_{\mathrm{i}}} \nabla_{\mathrm{e}_{\mathrm{i}}} \nabla_{\mathrm{e}_{\mathrm{j}}} \varphi \\
& +\sum_{\mathrm{i}, \mathrm{j}=1, \ldots, \mathrm{n}} \mathrm{e}_{\mathrm{j}}^{*} \odot \mathrm{le}_{\mathrm{i}} \nabla_{\mathrm{e}_{\mathrm{j}}} \nabla_{\mathrm{e}_{\mathrm{i}}} \varphi-\sum_{\mathrm{j}, \mathrm{r}=1, \ldots, \mathrm{n}} \mathrm{e}_{\mathrm{j}}^{*} \odot \mathrm{e}_{\mathrm{r}} \nabla \nabla \mathrm{e}_{\mathrm{j}} \mathrm{e}_{\mathrm{r}} \varphi \\
& =-\sum_{\mathrm{i}, \mathrm{j},=1, \ldots, \mathrm{n}} \Gamma_{\mathrm{ij}}^{\mathrm{i}} \nabla_{\mathrm{e}_{\mathrm{j}}} \varphi+\sum_{\mathrm{i}, \mathrm{r}=1, \ldots, \mathrm{n}} \mathrm{e}_{\mathrm{r}}{ }^{*} \bigodot_{1 \mathrm{e}_{\mathrm{i}}} \nabla \nabla \mathrm{e}_{\mathrm{i}} \mathrm{e}_{\mathrm{r}} \varphi \\
& -\sum_{\mathrm{i}=1, \ldots, \mathrm{n}} \nabla \mathrm{e}_{\mathrm{i}} \nabla_{\mathrm{e}_{\mathrm{i}}} \varphi-\sum_{\mathrm{i}, \mathrm{j}=1, \ldots, \mathrm{n}} \mathrm{e}_{\mathrm{j}}^{*} \odot \mathrm{e}_{\mathrm{i}} \nabla_{\mathrm{e}_{\mathrm{i}}} \nabla_{\mathrm{e}_{\mathrm{j}}} \varphi \\
& +\sum_{\mathrm{i}, \mathrm{j}=1, \ldots, \mathrm{n}} \mathrm{e}_{\mathrm{j}}{ }^{*} \odot \mathrm{le}_{\mathrm{i}} \nabla_{\mathrm{e}_{\mathrm{j}}} \nabla_{\mathrm{e}_{\mathrm{i}}} \varphi-\sum_{\mathrm{j}, \mathrm{r}=1, \ldots, \mathrm{n}} \mathrm{e}_{\mathrm{j}}^{*} \odot \mathrm{e}_{\mathrm{r}} \nabla \nabla \mathrm{e}_{\mathrm{j}} \mathrm{e}_{\mathrm{r}} \varphi
\end{aligned}
$$




$$
\begin{aligned}
& =\sum_{\mathrm{i}, \mathrm{j}=1, \ldots, \mathrm{n}} \Gamma_{\mathrm{ii}}^{\mathrm{j}} \nabla_{\mathrm{e}_{\mathrm{j}}} \varphi-\sum_{\mathrm{i},=1, \ldots, \mathrm{n}} \nabla_{\mathrm{e}_{\mathrm{i}}} \nabla_{\mathrm{e}_{\mathrm{i}}} \varphi \\
& -\sum_{\mathrm{i}, \mathrm{j}=1, \ldots, \mathrm{n}} \mathrm{e}_{\mathrm{j}}^{*} \odot \mathrm{le}_{\mathrm{i}}\left(\nabla_{\mathrm{e}_{\mathrm{i}}} \nabla \mathrm{e}_{\mathrm{j}} \varphi-\nabla \nabla \mathrm{e}_{\mathrm{i}} \mathrm{e}_{\mathrm{j}} \varphi\right) \\
& +\sum_{\mathrm{i}, \mathrm{j}=1, \ldots, \mathrm{n}} \mathrm{e}_{\mathrm{j}}^{*} \odot \mathrm{e}_{\mathrm{i}}\left(\nabla_{\mathrm{e}_{\mathrm{j}}} \nabla_{\mathrm{e}_{\mathrm{i}}} \varphi-\nabla \nabla \mathrm{e}_{\mathrm{j}} \mathrm{e}_{\mathrm{i}} \varphi\right) \\
& =-\sum_{\mathrm{i},=1, \ldots, \mathrm{n}}\left(\nabla \mathrm{e}_{\mathrm{i}} \nabla_{\mathrm{e}_{\mathrm{i}}} \varphi-\nabla \nabla \mathrm{e}_{\mathrm{i}} \mathrm{e}_{\mathrm{i}} \varphi\right) \\
& -\sum_{\mathrm{i}, \mathrm{j}=1, \ldots, \mathrm{n}} \mathrm{e}_{\mathrm{j}}^{*} \odot \mathrm{e}_{\mathrm{i}}\left(\nabla \mathrm{e}_{\mathrm{i}} \nabla_{\mathrm{e}_{\mathrm{j}}} \varphi-\nabla \nabla \mathrm{e}_{\mathrm{i}} \mathrm{e}_{\mathrm{j}} \varphi\right) \\
& +\sum_{\mathrm{i}, \mathrm{j}=1, \ldots, \mathrm{n}} \mathrm{e}_{\mathrm{j}}^{*} \odot \mathrm{e}_{\mathrm{i}}\left(\nabla \mathrm{e}_{\mathrm{j}} \nabla \mathrm{e}_{\mathrm{i}} \varphi-\nabla \nabla \mathrm{e}_{\mathrm{j}} \mathrm{e}_{\mathrm{i}} \varphi\right) \text {. }
\end{aligned}
$$

By Proposition 2.6, Theorem 2.3, (18), the definition of curvature and (20) we get the assertion.

Let us terminate the section with the remark that in the light of Theorem 2.3 our formula (21) is equivalent to the following classical Weitzenböck formula in the bundle of symmetric tensors on a Riemannian manifold

$$
\Delta^{\mathrm{s}}=-\operatorname{tr} \nabla \nabla-\mathfrak{R}
$$

where $\Re$ is Ricci type tensor defined in (20).

A proof of the classical Weitzenböck formula in form (22) can be find e.g. in [5] or [1].

\section{Conclusions}

The linear combinations of operators $\mathrm{d}^{\mathrm{s}}$ (defined by (7)), $a$ (defined by (13)) and two traces $\mathrm{Tr}$ and $\operatorname{tr}$ (defined by (11) and (12)), respectively) and their adjoins led to several interesting operators. The operators considered here are grad, div, or the important operators $-\operatorname{tr} \nabla \nabla$ or $\Delta^{\mathrm{s}}$. These operators, of course, do not complete the list of operators that can be studied. The possible examples are operators that arise by the process of removing the traces like the Ahlfors operator investigated in [11] or the conformal Killing operator in the bundle of symmetric tensors considered in [4] or [3]. We are going to continue investigation in this direction in a subsequent paper.

Finally notice that the Weitzenböck type formula (21) relates two differential operators on symmetric forms: - div grad and the Laplace operator. The geometric importance of the formula comes from the fact that the difference between these second order operators is an operator of order zero: the Ricci type tensor $R$ and that this tensor depends essentially on the curvature of $M$. In some particular cases of manifolds with the defined Ricci form $R$, this may be applied (with use of the classical Bochner technique) to determinate some geometrically important objects like conformal or harmonic tensor fields on a given manifold. The Killing, conformal Killing and trace free conformal Killing tensor, that constitute a subclass of the class of symmetric tensors considered in our paper, have an application in various problems of geometry, physics and tomography (see eg. $[3,12,13]$ ). 


\section{References}

[1] Balcerzak B., Pierzchalski A., Generalized gradients on Lie algebroids, Ann. Global Anal. Geom. 2013, 44(3), 319-337.

[2] Stepanov S.E., Mikes J., The spectral theory of the Yano rough Laplacian with some of its applications, Ann Global Anal. Geom. 2015, 48, 37-46.

[3] Heil K., Moroianu A., Semmelmann U., Killing and conformal Killing tensors, J. Geom. Phys. 2016, 106, 383-400.

[4] Reimann H.M., A rotation invariant differential equation for vector fields, Ann. Scuola Norm. Sup. Pisa 1982, 9, 160-174.

[5] Kimaczyńska A., The differential operators in the bundle of symmetric tensors on a Riemannian manifold, PhD thesis, Lódź University, Faculty of Mathematics and Computer Science, Lódź 2016, 64 p.

[6] Kimaczyńska A., Pierzchalski A., Elliptic operators in the bundle of symmetric tensors, Banach Center Publications (To appear).

[7] Sampson J.H., On a theorem of Chern, AMS 1973, 177, 141-153.

[8] Kobayashi S., Nomizu K., Foundations of differential geometry II, Interscience Publishers, New York - London 1969.

[9] Yu Y., The Index Theorem and the Heat Equation Method, World Scientific, Singapore - New Jersey - London - Hong Kong 2000.

[10] Kozłowski W., Pierzchalski A., Natural boundary value problems for weighted form Laplacians, Ann. Scuola Norm. Sup. Pisa Cl. Sci. 2008, (5), VII, 343-367.

[11] Pierzchalski A., Ricci curvature and quasiconformal deformations of a Riemannian manifold, Manuscripta Mathematica 1989, 66, 113-127.

[12] Dairbekov N.S., Sharafutdinov V.A., Conformal Killing symmetric tensor fields on Riemannian manifolds (Russian), Mat. Tr. 2010, 13, no. 1, 85-145; translation in Siberian Adv. Math. 2011, $21,1,1-41$.

[13] Stepanov S., Tsyganok I., Aleksandrova I., A remark on Laplacian operator which acts on symmetric tensors, arXiv:1411.1928. 